\title{
Between-row spacing and local accession on the yield and quality of garlic
}

\author{
Jamal-Ali Olfati*, Mohammad-Bagher Mahdieh-Najafabadi', Mohammad Rabiee²
}

\author{
'University of Guilan, Rasht, Islamic Republic of Iran \\ ${ }^{2}$ National Rice Research Institute, Rasht, Islamic Republic of Iran \\ Corresponding author, e-mail: jamalaliolfati@gmail.com
}

\begin{abstract}
Garlic is primarily grown for its cloves used mostly as a food flavoring condiment. Previous studies carried out on plant density indicate its direct influence on yield. Plant density depends on the genotype, environmental factors, cultural practices, etc. This study was established to determine the effects of different between-row spacing on growth, yield, and quality of four local accession of garlic. It was laid out on two-factorial Randomized Complete Block Design with three replications during two years. Four local accession of garlic (Langroud, Tarom, Tabriz and Hamedan) were culture in three between-rows spacing $(15,25$ and $35 \mathrm{~cm})$ during two years. The results of two cultivated years were different. Plant density changed when garlic cultured with different between row spacing. In present research plant yield increased when the lower between row spacing and high plant density were used but the yield improvement occurring at increased plant stand is offset by the reduction in bulb size and some quality indices such as total phenol and antioxidant which severely affects quality and market value, when garlic is produced for fresh market.
\end{abstract}

Keywords: harvesting index, bulb yield, antioxidant capacity, total phenol

\section{Espaçamento entre linhas e acessos locais na produtividade e qualidade do alho}

\section{Resumo}

O alho é cultivado principalmente por seus dentes e é usado principalmente como um condimento. Estudos anteriores realizados sobre densidade de plantas indicam a sua influência direta sobre o rendimento. A densidade de plantas depende do genótipo, fatores ambientais, práticas culturais, etc. Este estudo foi estabelecido para determinar os efeitos de diferentes espaçamentos entre linhas no crescimento, rendimento e qualidade de quatro acessos locais de alho. $\bigcirc$ delineamento foi blocos casualizados em esquema fatorial, com três repetições, durante dois anos. Quatro acessos locais de alho (Langroud, Tarom, Tabriz e Hamedan) foram cultivados em três espaçamentos entre linhas $(15,25$ e $35 \mathrm{~cm})$ durante dois anos. Os resultados de dois anos cultivados foram diferentes. A densidade de plantas foi alterada quando o alho foi cultivado em diferentes espaçamentos entre linhas. Na presente pesquisa a produtividade por planta aumentou quando o espaçamento entre linhas foi inferior e alta densidade de plantas foram utilizadas, mas a melhoria de rendimento ocorre melhor estande de plantas e redução no tamanho do bulbo e de alguns índices de qualidade, tais como fenóis totais e antioxidantes que afetam severamente a qualidade e valor de mercado, quando o alho é produzido para o mercado in natura.

Palavras-chave: índice de colheita, produção de bulbos, capacidade antioxidante, fenóis totais 


\section{Introduction}

Garlic is primarily grown for its cloves used mostly as a food flavoring condiment. But many producers in northern Iran cultivated this crop for its leaves to prepare different local dishes (Olfati et al., 2010). Garlic has the high contents of organosulphur antioxidant compounds (AbdelWahhab et al., 2012).

Previous studies carried out on plant density indicate its direct influence on different crop yield (Castille et al., 1996; Wang et al., 1997; Castellanos et al., 2004; Lima et al., 2012; Ibrahim, 2012; Zhang et al., 2012; Souza et al. 2013; Nyakudya and Stroosnijder, 2014). They indicated that although plant yield increased when the higher plant density were used but the yield improvement occurring at increased plant stand is offset by the reduction in bulb size which severely affects quality and market value, when garlic is produced for fresh market (Castellanos et al., 2004). Castillo et al. (1996) recommended handling plant densities from 140000 to 180000 plants/ha to ensure a good bulb diameter.

The photosynthetic apparatus performs photosynthesis, which involves the production of organic matter (carbohydrates, protein and fats) in organic or conventional production systems. The leaves are the most important part of the plant for photosynthetic activity. Photosynthesis depends on a large number of factors. Foremost among these is the chlorophyll content of the leaves, followed by the leaf surface area, which determines how much sunlight, will be absorbed by the plant. In addition, photosynthesis depends on the genotype (Moracevic, 2011). Leaf number per plant, leaf area per plant, and leaf area index (LAI), which represents a crop's total leaf area per unit area $\left(\mathrm{m}^{2} / \mathrm{ha}\right)$ are the most important factors in plant photosynthesis ability. Garlic develops 8 to 14 leaves. The photosynthetic apparatus of a cultivated plant can be acted upon via plant density (leaf number per unit area). Plant density depends on the genotype, environmental factors, cultural practices, etc. It should be noted that a thinner stand promotes the expression of an individual plant's potential, whereas denser stands are conducive to a greater expression of the plants' collective potential (crop potential). This has been noted by many authors, especially those working in the field of plant physiology. Many researchers have studied the effects of stand density on garlic. Data are also available for leaf number per plant, leaf area, and leaf area index. Studies have shown that there is no single, universal plant density that would be optimal for a garlic crop regardless of the soil and climatic conditions it is grown under.

Llosas and Fernandez (1984) argue that the ideal stand density for garlic is about 800,000 plants/ha. They note that this particular density is very high, but that it only negatively affects bulb uniformity. According to Lewis et al. (1995), garlic responds best to a plant population of 330,000 individuals/ha, as this density allows the plant in general and the leaves in particular to develop optimally. Results obtained by Muro et al. (2000) indicate that garlic leaf parameters (leaf number per plant, leaf area and leaf mass) reach their maximum values three weeks before the bulbs are harvested. Ahmad and labal (2002) studied plant densities of up to 2,000,000 plants/ha, but they achieved the best results with stands of moderate density (around 600,000 plants/ha).

There is evidence that garlic grows best when there are about 600,000 plants/ha (Morav $\square$ evi $\square$ et al., 2011). According to Karaye and Yakubu (2006), garlic plants become more luxuriant in thinner stands, while bulb yield increases at greater plant populations. The authors suggest that garlic should be grown in a thick stand to give priority to yield. Having studied plant populations between 330,000 and 1,300,000 plants per hectare, Kilgori et al. (2007a, b) found that garlic responded best to a stand of 600,000 plants/ha.

There is little information on the affects of plant density on garlic quality so the objective of this study was to determine the effects of different between-row spacing on growth, yield and quality of four local accession of garlic to reach maximum yield with the best quality.

\section{Materials and Methods}

The experiment was conducted at the National Rice Research Institute, Rasht, Iran, during 2008-2010. Table 1 refers to rainfall and temperature during experiment months in this region. It was laid out on factorial Randomized 
Complete Block Design $(4 \times 2)$ with three replications during two years. Four local accession of garlic (Langroud, Tarom, Tabriz and Hamedan) were culture in three between-rows spacing 115 , 25 and $35 \mathrm{~cm}$ ). All conventional cultural practices were adopted as recommended for this region (Mahdieh Najafabadi et al., 2012). The soil in the field plots was a sandy loam, pH 7.4, EC of 0.08 ds $\mathrm{cm}^{-1}$, and a $\mathrm{C} / \mathrm{N}$ ratio of 0.43 . Soil in the seedbeds was prepared by plowing and disking following local farm practices. The cloves were planted on November 5, 2008 and 2009, with a distance of $0.10 \mathrm{~m}$ between plants and harvested on 20 May 2009 and 2010 (Muro et al., 2000). The observations recorded during these studies were, bulb and neck diameter and their ratio (Forcing characteristics); bulb weight and yield were also recorded.

Table 1. Temperatures and rainfall at garlic planting site

\begin{tabular}{|c|c|c|c|c|c|c|c|c|c|c|c|c|c|c|}
\hline \multirow{2}{*}{ Month } & \multicolumn{2}{|c|}{ Nov. } & \multicolumn{2}{|c|}{ Dec. } & \multicolumn{2}{|c|}{ Jan. } & \multicolumn{2}{|c|}{ Feb. } & \multicolumn{2}{|c|}{ Mar. } & \multicolumn{2}{|c|}{ Apr. } & \multicolumn{2}{|c|}{ May. } \\
\hline & 2008 & 2009 & 2008 & 2009 & 2009 & 2010 & 2009 & 2010 & 2009 & 2010 & 2009 & 2010 & 2009 & 2010 \\
\hline Rainfall (mm) & 178.9 & 170.0 & 238.4 & 94.7 & 149.0 & 47.9 & 66.2 & 127.5 & 27.3 & 89.8 & 133.0 & 74.4 & 44.9 & 140.0 \\
\hline $\begin{array}{l}\text { Temperature } \\
\left({ }^{\circ} \mathrm{C}\right) \text { Max. }\end{array}$ & 17.4 & 18 & 14.1 & 15.0 & 9.6 & 14.3 & 12.8 & 10.2 & 15.9 & 13.7 & 16.8 & 16.2 & 22.2 & 21.0 \\
\hline $\begin{array}{l}\text { Temperature } \\
\left({ }^{\circ} \mathrm{C}\right) \text { Min. }\end{array}$ & 10.5 & 10.7 & 5.9 & 5.9 & 2.2 & 7.3 & 4.9 & 3.5 & 5.8 & 7.7 & 6.0 & 8.3 & 12.8 & 14.0 \\
\hline
\end{tabular}

Dry matter was determined by drying at $75 \pm 5^{\circ} \mathrm{C}$ until samples reached constant weight (AOAC, 1984). Total soluble solid contents (TSS) were determined by squeezing the tissue and placing one drop of juice from each sample into a refractometer (Atago HSR-500, Tokyo, Japan).

Methanol extracts of sample (1 $\mathrm{g}$ sample in $10 \mathrm{cc}$ methanol) were used for determination of total phenolics. Total phenolic content was evaluated by colorimetric analyses using Folin-Ciocaltave's phenol reagent (Singleton and Rossi, 1965; Hassan et al., 2013). The total phenolics content was expressed as mg galic acid equivalent/100 $\mathrm{g}$ of sample.

The free radical-scavenging activity against DPPH radical was evaluated with the methods of Leonge and Shui (2002) and Miliouskas et al. (2004) with minor modification. In the presence of an antioxidant, the purple color intensity of DPPH solution decays and the change of absorbance are followed spectrophotometrically at $517 \mathrm{~nm}$.

Measurement of total flavonoids was performed according to method Bozin et al. (2008) and Baghiani et al. (2010). Potassium was measured by flame photometery (Model AT-8000, Gold, Shanghai, China) according to Feitosa et al., (2013); Mg and P were measured with a UV/Vis spectrophotometer (Model 6405, Jenway, Essex, UK).
Data were subjected to analysis of variance in SAS (SAS, Inc., Cary, NC). If interactions were significant they were used to explain the data. If interactions were not significant means were separated with Tukey test.

\section{Results}

There are significant differences between years so we analyzed each year data separately.

First year: Interaction between local accessions and between-row spacing had affected biomass, bulb yield, bulb antioxidant capacity, total phenol, flavonoids and P content significantly (Table 2-4).

Between row spacing had affected total yield, biomass, bulb yield, neck diameter, cormlet weight, corm ash, total phenol and flavonoids content significantly (Table 2-4). There are also significant differences between local accession on total yield, biomass, bulb yield, harvesting index, number of leaves, neck diameter, corm diameter, corm diameter to neck diameter ratio, corm height, corm weight, number of cormlet, cormlet weight, corm dry matter, TSS, antioxidant capacity, flavonoids and $\mathrm{P}$ content (Table 2-4).

The $15 \mathrm{~cm}$ between row lead to the highest total yield and corm ash and the lowest corm diameter and cormlet weight (Table 5).

Tarom accession had the higher harvesting index, number of leaves, corm diameter, neck diameter to corm diameter ratio, cormlet weight and corm dry matter and 
Olfati et al. (2016) / Between-row spacing and local accession...

Table 2. ANOVA table of affects of between row spacing and local accessions on garlic yield and yield component.

\begin{tabular}{|c|c|c|c|c|c|c|c|c|c|}
\hline S.O.V. & d.f. & $\begin{array}{c}\text { Total yield } \\
\left(t \cdot h a^{-1}\right)\end{array}$ & $\begin{array}{c}\text { Biomass } \\
\left(t \cdot h a^{-1}\right)\end{array}$ & $\begin{array}{c}\text { Plant } \\
\text { weight (g) }\end{array}$ & $\begin{array}{l}\text { Corm } \\
\text { yield } \\
\left(t \cdot h a^{-1}\right)\end{array}$ & $\begin{array}{l}\text { Harvesting index } \\
\text { (Corm yield/ } \\
\text { Total yield) }\end{array}$ & $\begin{array}{l}\text { Corm } \\
\text { weight } \\
\text { (g) }\end{array}$ & $\begin{array}{l}\text { Number } \\
\text { of } \\
\text { cormlet }\end{array}$ & $\begin{array}{l}\text { Cormlet } \\
\text { weight } \\
\text { (g) }\end{array}$ \\
\hline \multicolumn{10}{|l|}{ First year } \\
\hline $\begin{array}{l}\text { Replication } \\
\text { (R) }\end{array}$ & 2 & $12.53 \mathrm{~ns}$ & $0.01 \mathrm{~ns}$ & $611.31 \mathrm{~ns}$ & $3.36 \mathrm{~ns}$ & $21.87 \mathrm{~ns}$ & 3208.98* & $5.26 \mathrm{~ns}$ & $13.44^{* *}$ \\
\hline $\begin{array}{l}\text { Between row } \\
\text { spacing (B) }\end{array}$ & 2 & $570.58^{* *}$ & $0.18^{*}$ & 4754.38ns & $69.96^{*}$ & $18.14 \mathrm{~ns}$ & $399.57 \mathrm{~ns}$ & $4.81 \mathrm{~ns}$ & $8.18^{*}$ \\
\hline $\mathrm{R} * \mathrm{~B}$ & 4 & 24.98 & 0.01 & 1961.06 & 8.07 & 40.56 & 266.35 & 3.92 & 0.49 \\
\hline $\begin{array}{l}\text { Local } \\
\text { accession (A) }\end{array}$ & 3 & $94.6^{* *}$ & $0.05^{* *}$ & $912.64 \mathrm{~ns}$ & $26.17^{* *}$ & $71.81^{* *}$ & $260.68^{*}$ & $66.39 * *$ & $56.5^{* *}$ \\
\hline $\mathrm{B}^{*} \mathrm{~A}$ & 6 & $10.05 \mathrm{~ns}$ & $0.003^{*}$ & $587.96 \mathrm{~ns}$ & $1.72 *$ & $4.26 \mathrm{~ns}$ & $69.43 \mathrm{~ns}$ & $3.40 \mathrm{~ns}$ & $1.04 \mathrm{~ns}$ \\
\hline Error & 18 & 3.85 & 0.001 & 527.01 & 0.44 & 4.53 & 80.12 & 7.78 & 2.06 \\
\hline C.V. (\%) & & 8.08 & 7.45 & 12.59 & 7.61 & 5.93 & 12.96 & 15.98 & 10.19 \\
\hline \multicolumn{10}{|l|}{ Second year } \\
\hline $\begin{array}{l}\text { Replication } \\
\text { (R) }\end{array}$ & 2 & $0.48 n s$ & $0.002 \mathrm{~ns}$ & $27.12 \mathrm{~ns}$ & $0.41 \mathrm{~ns}$ & $11.39 \mathrm{~ns}$ & $43.01 \mathrm{~ns}$ & $7.80 \mathrm{~ns}$ & $0.01 \mathrm{~ns}$ \\
\hline $\begin{array}{l}\text { Between row } \\
\text { spacing (B) }\end{array}$ & 2 & $101.38^{* *}$ & $0.05^{* *}$ & $27.43 n s$ & $21.79 * *$ & $56.13^{*}$ & $64.21 \mathrm{~ns}$ & $0.08 n s$ & $1.33 \mathrm{~ns}$ \\
\hline $\mathrm{R}^{*} \mathrm{~B}$ & 4 & 3.06 & 0.001 & 38.41 & 0.82 & 8.05 & 17.89 & 2.74 & 0.21 \\
\hline $\begin{array}{l}\text { Local } \\
\text { accession (A) }\end{array}$ & 3 & $4.78^{*}$ & $0.001 \mathrm{~ns}$ & $51.29 *$ & $0.87 n s$ & $28.75^{*}$ & $47.86^{*}$ & $8.22^{* *}$ & $0.03 \mathrm{~ns}$ \\
\hline$B^{*} A$ & 6 & $2.50 \mathrm{~ns}$ & $0.001 \mathrm{~ns}$ & $51.23^{*}$ & $0.38 n s$ & $29.93^{*}$ & $6.13 \mathrm{~ns}$ & $1.16 \mathrm{~ns}$ & $0.09 \mathrm{~ns}$ \\
\hline Error & 18 & 1.28 & 0.001 & 13.49 & 0.31 & 8.83 & 9.54 & 0.96 & 0.19 \\
\hline C.V. (\%) & & 15.83 & 17.53 & 9.24 & 15.49 & 5.83 & 12.75 & 9.94 & 17.20 \\
\hline
\end{tabular}

Table 3. ANOVA table of affects of between row spacing and local accessions on garlic vegetative characteristics.

\begin{tabular}{|c|c|c|c|c|c|c|c|c|}
\hline S.O.V. & d.f. & $\begin{array}{c}\text { Plant } \\
\text { height (\%) }\end{array}$ & $\begin{array}{l}\text { Number } \\
\text { of leaves }\end{array}$ & $\begin{array}{c}\text { Neck } \\
\text { diameter } \\
(\mathrm{cm})\end{array}$ & $\begin{array}{c}\text { Corm } \\
\text { diameter } \\
(\mathrm{cm})\end{array}$ & $\begin{array}{c}\text { Neck } \\
\text { diameter/corm } \\
\text { diameter }\end{array}$ & $\begin{array}{c}\text { Corm } \\
\text { height }(\mathrm{cm})\end{array}$ & $\begin{array}{c}\text { Cormlet } \\
\text { diameter }(\mathrm{cm})\end{array}$ \\
\hline \multicolumn{9}{|l|}{ First year } \\
\hline $\begin{array}{l}\text { Replication } \\
\text { (R) }\end{array}$ & 2 & $271.57 \mathrm{~ns}$ & $111.47 \mathrm{~ns}$ & $16.73 \mathrm{~ns}$ & $51.72 *$ & $0.42 \mathrm{~ns}$ & $271.87 \mathrm{~ns}$ & $26.58 \mathrm{~ns}$ \\
\hline $\begin{array}{l}\text { Between row } \\
\text { spacing (B) }\end{array}$ & 2 & $234.95 \mathrm{~ns}$ & $11.55 \mathrm{~ns}$ & $12.56 \mathrm{~ns}$ & $57.36^{*}$ & $0.41 \mathrm{~ns}$ & $117.33 n s$ & $1.47 \mathrm{~ns}$ \\
\hline $\mathrm{R}^{*} \mathrm{~B}$ & 4 & 62.34 & 32.64 & 7.24 & 5.62 & 0.48 & 46.04 & 4.55 \\
\hline $\begin{array}{l}\text { Local } \\
\text { accession (A) }\end{array}$ & 3 & $70.32 \mathrm{~ns}$ & $320.75^{* *}$ & $21.29 *$ & $88.32^{* *}$ & $2.27^{* *}$ & $110.32^{* *}$ & $9.11 \mathrm{~ns}$ \\
\hline$B^{*} A$ & 6 & $14.98 \mathrm{~ns}$ & $17.73 \mathrm{~ns}$ & $7.09 \mathrm{~ns}$ & $9.26 \mathrm{~ns}$ & $0.54 \mathrm{~ns}$ & $12.72 \mathrm{~ns}$ & $9.88 \mathrm{~ns}$ \\
\hline Error & 18 & 24.58 & 23.37 & 4.42 & 9.16 & 0.38 & 7.92 & 44.46 \\
\hline C.V. (\%) & & 5.76 & 31.13 & 12.10 & 4.99 & 17.19 & 6.36 & 10.86 \\
\hline \multicolumn{9}{|l|}{ Second year } \\
\hline $\begin{array}{l}\text { Replication } \\
\text { (R) }\end{array}$ & 2 & $87.61 \mathrm{~ns}$ & $30.71^{*}$ & $17.24 \mathrm{~ns}$ & $9.16 \mathrm{~ns}$ & $0.70^{*}$ & $11.58 \mathrm{~ns}$ & $4.83 \mathrm{~ns}$ \\
\hline $\begin{array}{l}\text { Between row } \\
\text { spacing (B) }\end{array}$ & 2 & $53.65 \mathrm{~ns}$ & $3.33 \mathrm{~ns}$ & $1.50 \mathrm{~ns}$ & $7.31 \mathrm{~ns}$ & $0.05 \mathrm{~ns}$ & $2.30 \mathrm{~ns}$ & $7.25^{*}$ \\
\hline $\mathrm{R}^{*} \mathrm{~B}$ & 4 & 19.10 & 2.81 & 2.79 & 13.11 & 0.09 & 3.48 & 0.84 \\
\hline $\begin{array}{l}\text { Local } \\
\text { accession (A) }\end{array}$ & 3 & $41.59 \mathrm{~ns}$ & $2.97 \mathrm{~ns}$ & $3.35 \mathrm{~ns}$ & $37.16^{* *}$ & $0.14 \mathrm{~ns}$ & $4.37 n s$ & $2.48 n s$ \\
\hline$B^{*} A$ & 6 & $13.95 \mathrm{~ns}$ & $7.29 \mathrm{~ns}$ & $1.98 \mathrm{~ns}$ & 13.38ns & $0.14 \mathrm{~ns}$ & $2.88 \mathrm{~ns}$ & $1.48 \mathrm{~ns}$ \\
\hline Error & 18 & 23.87 & 4.05 & 1.96 & 5.54 & 0.09 & 3.88 & 1.03 \\
\hline C.V. (\%) & & 5.87 & 16.85 & 9.25 & 5.52 & 10.44 & 6.23 & 7.12 \\
\hline
\end{tabular}


Table 3. Continue.

\begin{tabular}{|c|c|c|c|c|}
\hline S.O.V. & d.f. & $\begin{array}{l}\text { Cormlet width } \\
(\mathrm{cm})\end{array}$ & $\begin{array}{l}\text { Cormlet height } \\
(\mathrm{cm})\end{array}$ & $\begin{array}{l}\text { Corm height/ } \\
\text { corm diameter }\end{array}$ \\
\hline \multicolumn{5}{|l|}{ First year } \\
\hline Replication (R) & 2 & $11.2 \mathrm{~ns}$ & $15.52 \mathrm{~ns}$ & $0.03 \mathrm{~ns}$ \\
\hline Between row spacing (B) & 2 & $6.11 \mathrm{~ns}$ & $0.25 \mathrm{~ns}$ & $0.01 \mathrm{~ns}$ \\
\hline$R^{*} B$ & 4 & 6.86 & 2.86 & 0.01 \\
\hline Local accession (A) & 3 & $15.53 \mathrm{~ns}$ & $4.40 \mathrm{~ns}$ & $0.03 \mathrm{~ns}$ \\
\hline $\mathrm{B}^{*} \mathrm{~A}$ & 6 & $10.83 \mathrm{~ns}$ & $6.33 \mathrm{~ns}$ & $0.02 \mathrm{~ns}$ \\
\hline Error & 18 & 24.03 & 6.82 & 0.1 \\
\hline C.V. (\%) & & 15.97 & 8.91 & 12.37 \\
\hline \multicolumn{5}{|l|}{ Second year } \\
\hline Replication (R) & 2 & $0.95 \mathrm{~ns}$ & $5.64 \mathrm{~ns}$ & $0.02 \mathrm{~ns}$ \\
\hline Between row spacing (B) & 2 & $6.40^{*}$ & 14.39ns & $0.01 \mathrm{~ns}$ \\
\hline$R * B$ & 4 & 0.74 & 2.11 & 0.01 \\
\hline Local accession (A) & 3 & $0.38 \mathrm{~ns}$ & $3.39 \mathrm{~ns}$ & $0.01 \mathrm{~ns}$ \\
\hline $\mathrm{B}^{*} \mathrm{~A}$ & 6 & $0.65 \mathrm{~ns}$ & $0.55 \mathrm{~ns}$ & $0.01 \mathrm{~ns}$ \\
\hline Error & 18 & 1.39 & 1.18 & 0.005 \\
\hline C.V. (\%) & & 8.90 & 4.78 & 4.50 \\
\hline
\end{tabular}

Table 4. ANOVA table of affects of between row spacing and local accessions on garlic qualitative characteristics.

\begin{tabular}{|c|c|c|c|c|c|c|c|c|}
\hline S.O.V. & d.f. & $\begin{array}{c}\text { Antioxidant } \\
\text { (\% DPPH } \\
\text { reduction) }\end{array}$ & $\begin{array}{c}\text { Total phenol } \\
\text { (mg/100g } \\
\text { FW) }\end{array}$ & $\begin{array}{l}\text { Flavonoids } \\
\text { (mg/100g FW) }\end{array}$ & $\begin{array}{l}\text { Corm dry } \\
\text { matter (\%) }\end{array}$ & $\begin{array}{l}\text { Leaf dry } \\
\text { matter (\%) }\end{array}$ & $\begin{array}{l}\text { Corm } \\
\text { ash (\%) }\end{array}$ & $\begin{array}{c}\text { TSS } \\
\left({ }^{\circ} \text { Brix) }\right.\end{array}$ \\
\hline \multicolumn{9}{|l|}{ First year } \\
\hline Replication (R) & 2 & $38.13^{*}$ & $953.17^{*}$ & $444.11^{* *}$ & $31.97 \mathrm{~ns}$ & $26.71^{* *}$ & $2.20^{* *}$ & $7.36 \mathrm{~ns}$ \\
\hline $\begin{array}{l}\text { Between row } \\
\text { spacing (B) }\end{array}$ & 2 & $1.56 \mathrm{~ns}$ & $3822.88^{* *}$ & $1916.86^{* *}$ & $0.02 n s$ & $2.11 \mathrm{~ns}$ & $1.65^{*}$ & $0.001 \mathrm{~ns}$ \\
\hline $\mathrm{R} * \mathrm{~B}$ & 4 & 2.96 & 59.89 & 1.90 & 6.26 & 0.80 & 0.1 & 1.46 \\
\hline Local accession (A) & 3 & $23.60^{* *}$ & $27.66 \mathrm{~ns}$ & $587.44^{* *}$ & $48.32^{* *}$ & $0.33 n s$ & $0.92 \mathrm{~ns}$ & $10.87^{* *}$ \\
\hline $\mathrm{B}^{*} \mathrm{~A}$ & 6 & 5.23* & $454.04^{* *}$ & $627.82^{* *}$ & $1.42 \mathrm{~ns}$ & $0.53 \mathrm{~ns}$ & $0.75 n s$ & $0.34 \mathrm{~ns}$ \\
\hline Error & 18 & 1.33 & 26.63 & 8.53 & 2.20 & 1.08 & 0.3 & 0.51 \\
\hline C.V. (\%) & & 11.15 & 13.61 & 3.15 & 4.99 & 10.68 & 16.41 & 2.76 \\
\hline \multicolumn{9}{|l|}{ Second year } \\
\hline Replication (R) & 2 & $0.58 \mathrm{~ns}$ & $281.33^{*}$ & $576.33^{*}$ & $153.68^{*}$ & $1.42^{*}$ & $0.52 \mathrm{~ns}$ & $51.99 \mathrm{~ns}$ \\
\hline $\begin{array}{l}\text { Between row } \\
\text { spacing (B) }\end{array}$ & 2 & $5.84 \mathrm{~ns}$ & $113.65 \mathrm{~ns}$ & $15.25 \mathrm{~ns}$ & $39.21 \mathrm{~ns}$ & $0.80 \mathrm{~ns}$ & $0.33 n s$ & $11.49 \mathrm{~ns}$ \\
\hline $\mathrm{R}^{*} \mathrm{~B}$ & 4 & 3.58 & 22.67 & 81.58 & 9.32 & 0.19 & 1.85 & 11.80 \\
\hline Local accession (A) & 3 & $15.82^{*}$ & $1148.09 * *$ & $1711.48 \mathrm{~ns}$ & $1.34 \mathrm{~ns}$ & $2.03 \mathrm{~ns}$ & $3.01 \mathrm{~ns}$ & $0.61 \mathrm{~ns}$ \\
\hline $\mathrm{B}^{*} \mathrm{~A}$ & 6 & $17.62^{* *}$ & $1145.19^{* *}$ & $628.07 \mathrm{~ns}$ & $4.73 \mathrm{~ns}$ & $0.28 n s$ & $0.85 n s$ & $3.27 n s$ \\
\hline Error & 18 & 4.24 & 105.50 & 665.87 & 4.77 & 0.74 & 1.07 & 5.62 \\
\hline C.V. (\%) & & 11.39 & 15.55 & 15.28 & 6.70 & 8.84 & 16.45 & 9.17 \\
\hline
\end{tabular}


Table 4. Continue.

\begin{tabular}{|c|c|c|c|c|}
\hline S.O.V. & d.f. & $P(\mathrm{mg} / 100 \mathrm{~g} \mathrm{FW})$ & $\mathrm{K}(\mathrm{mg} / 100 \mathrm{~g} \mathrm{FW})$ & $\mathrm{Mg}(\mathrm{mg} / 100 \mathrm{~g} \mathrm{FW})$ \\
\hline \multicolumn{5}{|l|}{ First year } \\
\hline Replication (R) & 2 & $1889.06 \mathrm{~ns}$ & $372.76 \mathrm{~ns}$ & $482.71^{*}$ \\
\hline Between row spacing (B) & 2 & $2278.81 \mathrm{~ns}$ & $2124.58 \mathrm{~ns}$ & $5.35 \mathrm{~ns}$ \\
\hline$R * B$ & 4 & 387.64 & 4052.41 & 48.30 \\
\hline Local accession (A) & 3 & $1200.14^{* *}$ & $13404.44 n s$ & 99.70ns \\
\hline B*A & 6 & $899.35^{* *}$ & $2573.12 \mathrm{~ns}$ & $45.47 \mathrm{~ns}$ \\
\hline Error & 18 & 186.03 & 6646.63 & 97.12 \\
\hline C.V. (\%) & & 10.89 & 21.81 & 14.09 \\
\hline \multicolumn{5}{|l|}{ Second year } \\
\hline Replication (R) & 2 & $45.32 \mathrm{~ns}$ & $15442.56 \mathrm{~ns}$ & $768.79 *$ \\
\hline Between row spacing (B) & 2 & $610.34^{* *}$ & $28890.73^{*}$ & $362.48^{*}$ \\
\hline$R^{*} B$ & 4 & 16.25 & 2606.24 & 48.40 \\
\hline Local accession (A) & 3 & $211.82^{*}$ & $649.45 \mathrm{~ns}$ & $39.68 \mathrm{~ns}$ \\
\hline $\mathrm{B}^{*} \mathrm{~A}$ & 6 & $222.01^{* *}$ & 1944.68ns & $42.49 \mathrm{~ns}$ \\
\hline Error & 18 & 49.00 & 4203.18 & 61.47 \\
\hline C.V. (\%) & & 5.87 & 16.18 & 10.04 \\
\hline
\end{tabular}

Table 5. Effects of between row spacing on total yield, corm diameter, cormlet weight and corm ash in first year and total yield, biomass, corm yield, cormlet diameter, cormlet width, $\mathrm{K}$ and $\mathrm{Mg}$ in second year

\begin{tabular}{|c|c|c|c|c|c|c|c|}
\hline \multicolumn{8}{|c|}{ First year } \\
\hline $\begin{array}{l}\text { Between } \\
\text { row spacing }\end{array}$ & $\begin{array}{c}\text { Total yield } \\
\left(t \cdot h a^{-1}\right)\end{array}$ & $\begin{array}{l}\text { Corm } \\
\text { diameter } \\
(\mathrm{cm})\end{array}$ & $\begin{array}{c}\text { Cormlet } \\
\text { weight (g) }\end{array}$ & $\underset{(\%)}{\text { Corm }}$ & & & \\
\hline 15 & $31.25 a$ & $58.09 b$ & $6.37 b$ & $3.75 a$ & & & \\
\hline 25 & $24.09 a b$ & $62.08 a$ & $6.94 b$ & $3.03 b$ & & & \\
\hline 35 & $17.47 \mathrm{~b}$ & $61.64 a$ & $7.99 a$ & $3.22 \mathrm{~b}$ & & & \\
\hline \multicolumn{8}{|c|}{ Second year } \\
\hline $\begin{array}{l}\text { Between } \\
\text { row spacing }\end{array}$ & $\begin{array}{c}\text { Total yield } \\
\left(t \cdot h a^{-1}\right)\end{array}$ & $\begin{array}{l}\text { Biomass } \\
\left(t \cdot \mathrm{ha}^{-1}\right)\end{array}$ & $\begin{array}{c}\text { Corm yield } \\
\left(t \cdot h a^{-1}\right)\end{array}$ & $\begin{array}{c}\text { Cormlet } \\
\text { diameter } \\
(\mathrm{cm})\end{array}$ & $\begin{array}{c}\text { Cormlet } \\
\text { width }(\mathrm{cm})\end{array}$ & $\underset{F(m)}{(m g / 100 ~ g}$ & $\begin{array}{c}M g(\mathrm{mg} / 100 \\
g \text { FM) }\end{array}$ \\
\hline 15 & $10.21 a$ & $0.22 a$ & $4.95 a$ & $13.32 b$ & $12.46 \mathrm{~b}$ & $442.61 \mathrm{a}$ & $83.7 a$ \\
\hline 25 & $6.81 \mathrm{ab}$ & $0.14 b$ & $3.54 a b$ & $14.71 a$ & $13.91 \mathrm{a}$ & $359.12 b$ & $72.72 b$ \\
\hline 35 & $4.43 \mathrm{~b}$ & $0.09 \mathrm{~b}$ & $2.26 \mathrm{~b}$ & $14.62 \mathrm{ab}$ & $13.33 a b$ & $356.2 b$ & $77.81 \mathrm{ab}$ \\
\hline
\end{tabular}

the lowest corm height and number of cormlet while the highest total yield and neck diameter was obtained from Hamedan and Langroud accessions respectively (Table 6).

The highest biomass, total yield, total phenol and flavonoids were obtained when corms cultured with 15 between rows. The highest antioxidant capacity of Hamedan and Langroud accession were obtained from 25 between row spacing while for Tarom and Tabriz the highest antioxidant capacity were obtained from $15 \mathrm{~cm}$ between row spacing. The highest $\mathrm{p}$ content for all accessions were obtained from $15 \mathrm{~cm}$ between row spacing except Tabriz accession that the highest $P$ content was related to $25 \mathrm{~cm}$ between row spacing (Table 7). 
Table 6. Effects of local accessions on total yield, harvesting index, number of leaves, neck diameter, corm diameter, neck diameter/corm diameter, corm height, corm weight, number of cormlet, cormlet weight, corm dry matter and TSS in first year and total yield, corm diameter, corm weight, number of cormlet in second year

\begin{tabular}{|c|c|c|c|c|c|c|c|c|}
\hline \multicolumn{9}{|c|}{ First year } \\
\hline $\begin{array}{l}\text { Local } \\
\text { accession }\end{array}$ & $\begin{array}{c}\text { Total yield } \\
\left(t \cdot h a^{-1}\right)\end{array}$ & $\begin{array}{l}\text { Harvesting index } \\
\text { (Corm yield/ } \\
\text { total yield) }\end{array}$ & $\begin{array}{l}\text { Number of } \\
\text { leaves }\end{array}$ & $\begin{array}{c}\text { Neck } \\
\text { diameter } \\
\text { (cm) }\end{array}$ & $\begin{array}{l}\text { Corm } \\
\text { diameter } \\
(\mathrm{cm})\end{array}$ & $\begin{array}{c}\text { Neck } \\
\text { diameter/corm } \\
\text { diameter }\end{array}$ & $\begin{array}{l}\text { Corm } \\
\text { height } \\
(\mathrm{cm})\end{array}$ & $\begin{array}{l}\text { Corm } \\
\text { weight } \\
\text { (g) }\end{array}$ \\
\hline Hamedan & $26.54 a$ & $36.94 a b$ & $19 a b$ & $17.79 a b$ & $62.88 a b$ & $3.74 a b$ & $46.31 a$ & $75.51 a$ \\
\hline Tarom & $25.62 a$ & $39.26 a$ & $22.09 a$ & $15.12 b$ & $63.7 a$ & $4.24 a$ & $39.02 b$ & $71.55 a$ \\
\hline Langroud & $25.46 a$ & $34.79 \mathrm{bc}$ & $11.41 \mathrm{bc}$ & $18.60 a$ & $58.19 \mathrm{bc}$ & $3.17 b$ & $45.64 a$ & $64.79 a$ \\
\hline Tabriz & $19.46 \mathrm{~b}$ & $32.69 \mathrm{c}$ & $9.63 c$ & $17.96 a$ & $57.63 c$ & $3.22 a b$ & $46.05 a$ & $64.49 a$ \\
\hline \multicolumn{9}{|c|}{ First year } \\
\hline $\begin{array}{l}\text { Local } \\
\text { accession }\end{array}$ & $\begin{array}{c}\text { Number of } \\
\text { cormlet }\end{array}$ & $\begin{array}{l}\text { Cormlet weight } \\
\text { (g) }\end{array}$ & $\begin{array}{l}\text { Corm dry } \\
\text { matter (\%) }\end{array}$ & $\underset{\left({ }^{\circ} \text { Brix }\right)}{\text { Corm TSS }}$ & & & & \\
\hline Hamedan & $12.00 a$ & $6.69 \mathrm{~b}$ & $30.19 b$ & $26.00 \mathrm{~b}$ & & & & \\
\hline Tarom & $6.67 b$ & $10.76 a$ & $32.83 a$ & $27.24 a$ & & & & \\
\hline Langroud & $11.93 a$ & $5.57 b$ & $27.69 \mathrm{~b}$ & $24.81 b$ & & & & \\
\hline Tabriz & $12.33 a$ & $5.39 b$ & $28.27 \mathrm{~b}$ & $25.08 b$ & & & & \\
\hline \multicolumn{9}{|c|}{ Second year } \\
\hline $\begin{array}{l}\text { Local } \\
\text { accession }\end{array}$ & $\begin{array}{c}\text { Total yield } \\
\left(t \cdot h a^{-1}\right)\end{array}$ & $\begin{array}{c}\text { Corm diameter } \\
(\mathrm{cm})\end{array}$ & $\begin{array}{l}\text { Corm } \\
\text { weight (g) }\end{array}$ & $\begin{array}{l}\text { Number } \\
\text { of cormlet } \\
(\mathrm{cm})\end{array}$ & & & & \\
\hline Hamedan & $7.70 a$ & $42.07 a b$ & $25.05 a b$ & $9.36 a b$ & & & & \\
\hline Tarom & $7.85 a$ & $40.04 \mathrm{~b}$ & $21.27 \mathrm{~b}$ & $8.67 b$ & & & & \\
\hline Langroud & $6.43 a$ & $44.75 a$ & $26.76 a$ & $10.92 a$ & & & & \\
\hline Tabriz & $6.62 a$ & $43.58 a b$ & $23.86 a b$ & $10.22 a b$ & & & & \\
\hline
\end{tabular}

Second year: Interaction between local accessions and between-row spacing had affected plant weight, harvesting index, bulb antioxidant capacity, total phenol, and P content significantly (Table 2-4). Between row spacing had affected total yield, biomass, and bulb yield, harvesting index, cormlet diameter, cormlet width, $\mathrm{P}, \mathrm{K}$ and $\mathrm{Mg}$ content of bulb significantly (Table 5). There are also significant differences between local accession on total yield, plant weight, harvesting index, corm weight, number of cormlet, antioxidant capacity, flavonoids and $\mathrm{P}$ content (Table 6). $15 \mathrm{~cm}$ between row lead to the highest total yield, biomass, bulb yield, $\mathrm{K}$ and $\mathrm{Mg}$ and the lowest cormlet diameter and width (Table 5). Langroud accession was the best accession in second year according to all significant characteristics (Table 6). The highest plant weight of Tarom and Langroud accession were obtained from $35 \mathrm{~cm}$ between row spacing while for Hamedan and Tabriz the highest plant weight was obtained from 15 and $25 \mathrm{~cm}$ between rows spacing respectively (Table 7 ).

The highest harvesting index for all accessions were obtained from $25 \mathrm{~cm}$ between row spacing except Tabriz accession that the highest harvesting index was related to $35 \mathrm{~cm}$ between row spacing (Table 7). The highest antioxidant capacity of Hamedan, Tabriz and Tarom accession were obtained from 15 $\mathrm{cm}$ between row spacing while for Langroud accession the highest antioxidant capacity were obtained from $25 \mathrm{~cm}$ between row spacing (Table 7). The highest total phenol of Tabriz and Langroud accessions were obtained from $35 \mathrm{~cm}$ between row spacing while for Hamedan and Tarom accessions the highest phenol content were obtained from 25 and $15 \mathrm{~cm}$ between rows spacing respectively (Table 7 ). The highest $\mathrm{p}$ content for all accessions was obtained from 35 $\mathrm{cm}$ between row spacing (Table 7). 
Table 7. Effects of local accessions and between row spacing interaction on biomass, bulb yield, antioxidant capacity, total phenol, flavonoids and $\mathrm{P}$ in first year and plant weight, harvesting index, antioxidant, total phenol and $\mathrm{P}$ in second year.

\begin{tabular}{|c|c|c|c|c|c|c|c|c|c|}
\hline \multicolumn{10}{|c|}{ First year } \\
\hline $\begin{array}{l}\text { Local } \\
\text { accession }\end{array}$ & $\begin{array}{l}\text { Between row } \\
\text { spacing }\end{array}$ & $\begin{array}{l}\text { Biomass } \\
\left(t \cdot h a^{-1}\right)\end{array}$ & $\begin{array}{c}\text { Corm yield } \\
\left(t \cdot h a^{-1}\right)\end{array}$ & \multicolumn{2}{|c|}{$\begin{array}{c}\text { Antioxidant } \\
\text { capacity (\% } \\
\text { DPPH reduction) }\end{array}$} & \multicolumn{2}{|c|}{$\begin{array}{l}\text { Total phenol } \\
\text { (mg/100g } \\
\text { FM) }\end{array}$} & $\begin{array}{l}\text { Flavonoids } \\
\text { (mg/100g } \\
\text { FM) }\end{array}$ & $\stackrel{P}{(m g / 100 g ~ F M) ~}$ \\
\hline Hamedan & 15 & $0.6 \pm 0.06$ & $12.18 \pm 1.42$ & \multicolumn{2}{|c|}{$3.31 \pm 1.89$} & \multicolumn{2}{|c|}{$66.51 \pm 5.79$} & $108.67 \pm 8.50$ & $146.6 \pm 13.54$ \\
\hline Hamedan & 25 & $0.46 \pm 0.04$ & $10.62 \pm 1.55$ & \multicolumn{2}{|c|}{$5.71 \pm 1.82$} & \multicolumn{2}{|c|}{$20.91 \pm 8.01$} & $107.33 \pm 7.51$ & $92.22 \pm 10.87$ \\
\hline Hamedan & 35 & $0.31 \pm 0.02$ & $6.54 \pm 0.58$ & \multicolumn{2}{|c|}{$4.72 \pm 2.18$} & \multicolumn{2}{|c|}{$31.84 \pm 7.63$} & $83.67 \pm 10.69$ & $128.67 \pm 13.08$ \\
\hline Tarom & 15 & $0.61 \pm 0.1$ & $12.36 \pm 1.82$ & \multicolumn{2}{|c|}{$4.39 \pm 2.08$} & \multicolumn{2}{|c|}{$55.57 \pm 8.01$} & $92.33 \pm 5.51$ & $167.47 \pm 11.2$ \\
\hline Tarom & 25 & $0.45 \pm 0.03$ & $9.77 \pm 1.1$ & \multicolumn{2}{|c|}{$3.41 \pm 1.89$} & \multicolumn{2}{|c|}{$24.64 \pm 8.43$} & $74.33 \pm 5.51$ & $127.55 \pm 14.22$ \\
\hline Tarom & 35 & $0.38 \pm 0.02$ & $8.07 \pm 0.75$ & \multicolumn{2}{|c|}{$3.38 \pm 1.84$} & \multicolumn{2}{|c|}{$29.71 \pm 16.03$} & $78 \pm 3$ & $131.91 \pm 14.32$ \\
\hline Langroud & 15 & $0.53 \pm 0.05$ & $11.8 \pm 1.16$ & \multicolumn{2}{|c|}{$8.14 \pm 1.82$} & \multicolumn{2}{|c|}{$59.31 \pm 8.21$} & $80.33 \pm 5.13$ & $132.26 \pm 14.86$ \\
\hline Langroud & 25 & $0.43 \pm 0.05$ & $9.17 \pm 1.62$ & \multicolumn{2}{|c|}{$8.19 \pm 2.09$} & \multicolumn{2}{|c|}{$48.37 \pm 8.05$} & $118.33 \pm 3.51$ & $98.65 \pm 37.48$ \\
\hline Langroud & 35 & $0.27 \pm 0.01$ & $5.76 \pm 0.52$ & \multicolumn{2}{|c|}{$5.73 \pm 4.02$} & \multicolumn{2}{|c|}{$9.44 \pm 7.2$} & $77.33 \pm 4.51$ & $126.07 \pm 24.11$ \\
\hline Tabriz & 15 & $0.4 \pm 0.13$ & $7.77 \pm 2.62$ & \multicolumn{2}{|c|}{$7.38 \pm 1.53$} & \multicolumn{2}{|c|}{$52.11 \pm 7.69$} & $114.67 \pm 3.51$ & $110.65 \pm 9.16$ \\
\hline Tabriz & 25 & $0.32 \pm 0.06$ & $6.67 \pm 1.46$ & \multicolumn{2}{|c|}{$4.62 \pm 2.25$} & \multicolumn{2}{|c|}{$25.17 \pm 16.09$} & $103.33 \pm 10.5$ & $5 \quad 128.33 \pm 26.27$ \\
\hline Tabriz & 35 & $0.21 \pm 0.05$ & $4.52 \pm 0.65$ & \multicolumn{2}{|c|}{$6.5 \pm 1.4$} & \multicolumn{2}{|c|}{$31.31 \pm 16.21$} & $73.33 \pm 5.51$ & $113.1 \pm 18.76$ \\
\hline \multicolumn{10}{|c|}{ second year } \\
\hline $\begin{array}{l}\text { Local } \\
\text { accession }\end{array}$ & $\begin{array}{l}\text { Between } \\
\text { row spacing }\end{array}$ & $\begin{array}{c}\text { Plant } \\
\text { weight (g) }\end{array}$ & \multicolumn{2}{|c|}{$\begin{array}{l}\text { Harvesting index } \\
\text { (corm yield/total } \\
\text { yield) }\end{array}$} & $\begin{array}{l}\text { Antioxi } \\
\text { capacity } \\
\text { reduc }\end{array}$ & $\begin{array}{l}\text { idant } \\
\text { (\% DPPH } \\
\text { tion) }\end{array}$ & \multicolumn{2}{|c|}{$\begin{array}{c}\text { Total phenol } \\
\text { (mg/100g FM) }\end{array}$} & $P(\mathrm{mg} / 100 \mathrm{~g} F M)$ \\
\hline Hamedan & 15 & $40.81 \pm 5.29$ & $48.2 \pm 1.52$ & & $7.95 \pm 0.28$ & & 9.44 & $\pm 3 . .2$ & $113.55 \pm 1.84$ \\
\hline Hamedan & 25 & $43.63 \pm 2.9$ & $47.83 \pm 1.83$ & & $4.58 \pm 1.63$ & & 43.5 & $7 \pm 10.44$ & $116.95 \pm 5.36$ \\
\hline Hamedan & 35 & $38.67 \pm 5.53$ & $49.32 \pm 4.25$ & & $4.05 \pm 1.15$ & & 34.2 & $4 \pm 13.88$ & $112.43 \pm 2.29$ \\
\hline Tarom & 15 & $35.27 \pm 2.34$ & $48.71 \pm 2.2$ & & $7.3 \pm 3.64$ & & 54.2 & $4 \pm 10.4$ & $102.33 \pm 7.27$ \\
\hline Tarom & 25 & $38.78 \pm 0.96$ & $52.88 \pm 4.07$ & & $3.39 \pm 1.09$ & & 43.31 & $31 \pm 5.21$ & $121.86 \pm 4.05$ \\
\hline Tarom & 35 & $41.72 \pm 0.45$ & $53.44 \pm 2.78$ & & $9.07 \pm 0.21$ & & 17.97 & $7 \pm 3.61$ & $120.93 \pm 3.65$ \\
\hline Langroud & 15 & $44.98 \pm 6.82$ & $50.9 \pm 1.5$ & & $3.09 \pm 1.79$ & & 35.8 & $34 \pm 18.81$ & $109.68 \pm 7.67$ \\
\hline Langroud & 25 & $39.90 \pm 1.01$ & $50.9 \pm 1.43$ & & $2.74 \pm 1.02$ & & 35.8 & $34 \pm 2.4$ & $132.62 \pm 10.87$ \\
\hline Langroud & 35 & $36.75 \pm 7.45$ & $51.09 \pm 4.34$ & & $7.68 \pm 2.65$ & & $5.44 t$ & $4 \pm 2.88$ & $125.2 \pm 9.13$ \\
\hline Tabriz & 15 & $44.64 \pm 3.17$ & $51.74 \pm 1.44$ & & $4.36 \pm 1$ & & 54.77 & $77 \pm 2.81$ & $121.04 \pm 8.43$ \\
\hline Tabriz & 25 & $31.44 \pm 4.03$ & $59.72 \pm 2.58$ & & $3.01 \pm 2.4$ & & 63.31 & $31 \pm 17.2$ & $116 \pm 2.94$ \\
\hline Tabriz & 35 & $40.5 \pm 4.74$ & $47.27 \pm 4.71$ & & $6.15 \pm 3.06$ & & 33.71 & $71 \pm 12.01$ & $138.3 \pm 7.67$ \\
\hline
\end{tabular}

\section{Discussion}

As mention above, the results of two cultivated years were different. Garlic is a sensitive plant to water and high temperature stress (Miko et al., 2000). In first year the higher rainfall $(658.8 \mathrm{~mm}$ ) in comparison to second year (574.3 mm) and lower mean temperature (10.7 $\left.{ }^{\circ} \mathrm{C}\right)$ compaired to second year $\left(11.4^{\circ} \mathrm{C}\right)$ lead to better results.

Plant density changed when garlic cultured with different between row spacing. Previous studies carried out on plant density indicate its direct influence on yield (Castille et al., 1996; Castellanos et al., 2004). In present research plant yield increased when the lower between row spacing and high plant density were used but the yield improvement occurring at increased plant stand is offset by the reduction in bulb size and some quality indices such as total phenol and antioxidant which severely affects quality and market value, when garlic is produced for fresh market (Castellanos et al., 2004). However different local accessions differently response to between row spacing.

The number of leaves the most important part of the plant for photosynthetic activity per plant doesn't changed in each accession but their size increased by increasing between row spacing. In addition, photosynthesis depends on the genotype (Moracevic, 2011). Genotypes which had the higher leaves produce bigger corm. In other hand by increasing between row spacing and leaves surface bigger corm were harvested. Similar to previous research our results indicated that there is no single, universal plant density that would be optimal for a garlic crop regardless of the soil and climatic conditions it is grown under. 


\section{Conclusions}

Moderate between row spacing and plant density for fresh market garlic and low between row spacing and high plant density for higher yield is proposed according our results. More work is needed to select best culturing system for each variety and environment and select characteristics that are stable in different environment.

\section{References}

Abdel-Wahhab, K.G.E., Khadrawy, Y.A., Mannaa, F.A.E. 2012. Aged garlic extract enhances paraoxonase 1 activity and suppress oxidative stress in CCl4- intoxicated rats. Comunicata Scientiae 3: 55-63.

Ahmad, S., lqbal, J. 2002. Optimizing plant density cum weed control method for puccinial rust management and yield in garlic. Asian Journal of Plant Science 1: 197-198.

AOAC. Association of Official Analytical Chemists. 1984. Officials methods of analyses, $14^{\text {th }}$ ed. Arlington, VA.

Baghiani, A., Boumerfeg, S., Belkhiri, F., Khennouf, S., Charef, N., Harzallah, D., Arrar, L., Abdel M.A. 2010. Antioxidant and radical scavenging properties of Carthamus caeruleus L. extracts grow wild in Algeria flora. Comunicata Scientiae 1: 128-136.

Bozin, B., Mimica-Dukic, N., Samojlik, I., Goran, A., Igic, R., 2008. Phenolics as antioxidants in garlic (Allium sativum L.). Food chemistry 111: 925-929.

Castellanos, J.Z., Vargas-Tapia, P., Ojodeagua, J.L., Hoyos, G. 2004. Garlic productivity and profitability as affected by seed clove size, planting density and planting method. HortScience 39: 1272-1277.

Castillo, J.E., Lopez-Bellido, L., Fernandez, E.J., Lopez, F.J. 1996. Influence of planting geometry on growth, yield and quality of rain fed garlic (Allium sativum L.) cultivated under Mediterranean conditions. Journal of Horticultural Science 71: 867-879.

Lima, C.F., Arnhold, E., Araujo, B.L., Oliveira, G.H.F., Oliveira Junior, E.A. 2012. Evaluation of maize hybrids under three densities in the agricultural frontier in Maranhão State, Brazil. Comunicata Scientiae 3: 30-34.

Souza, R.S., Filho, P.S.V., Scapim, C.A., Marques, O.J., Queiroz, D.C., Okumura, R.S. 2013. Yield elements of sweet corn in different population densities. Comunicata Scientiae 4: 285-292.

Feitosa, H.O., Farias, G.C., Junior, R.J.C.S., Ferreira,
F.J., Filho, F.L.A., Lacerda, C.F. 2013. Influence of potassium fertilization and borácica performance Sunflower. Comunicata Scientiae 4: 302-307.

Hassan, N.S., Abdel-Wahhab, K.G., Khadrawy, Y.A., El-Nekeety, A.A., Mannaa, F.A., AbdelWahhab, M.A. 2013. Evaluation of radical scavenging properties and the protective role of papaya fruits extracts against oxidative stress in rats fed aflatoxin-contaminated diet. Comunicata Scientiae 4: 43-57.

Ibrahim, H.M. 2012. Response of some sunflower hybrids to different levels of plant density. APCBEE Procedia 4: 175-182.

Karaye, A.K., Yakubu, A.I. 2006. Influence of intrarow spacing and mulching on weed growth and bulb yield of garlic (Allium sativum L.) in Sokoto, Nigeria. African Journal of Biotechnology 5: 260264.

Kilgori, M.J., Magaji, M.D., Yakubu, A.I. 2007 . Effect of plant spacing and date of planting on yield of two garlic (Allium sativum L.) cultivars in Sokoto, Nigeria. American-Eurasian Journal of Agriculture and Environmental Science 2: 153157.

Kilgori, M.J., Magaji, M.D., Yakubu, A.I. $2007 b$. Productivity of two garlic (Allium sativum L.) cultivars as affected by different levels of nitrogenous and phosphorous fertilizers in Sokoto, Nigeria. American-Eurasian Journal of Agriculture and Environmental Science 2: 158-162.

Leong, L.P., Shui, G. 2002. An investigation of antioxidant capacity of fruits in Singapore markets. Food Chemistry, 76: 69-75.

Lewis, L.A., Ojeda, D.L., Salazar, M.O., Campbell, R.J. 1995. Effect of population density on growth, development and yield of garlic (Allium sativum L.) Cv. Vietnamita. Procedure of Interamerican Society of Tropical Horticulture 39: 23-26.

Llosas, C.N., Fernandez, A.M. 1984. Influence of planting density on growth, development and yields of garlic (Allium sativum L.). Centro Agricola 11:17-26.

Mahdieh Najafabadi, M.B., Peyvast, Gh., Hassanpour Asil, M., Olfati, J.A., Rabiee, M. 2012. mulching effects on the yield and quality of garlic as second crop in rice fields. International Journal of Plant Production 6: 279-290.

Miko, S., Ahmed, M.K., Amans, E.B., Falaki, A.M., llyas, N. 2000. Effects of levels of nitrogen, phosphorus and irrigation interval, on the performance and quality of garlic (Allium sativum L.). Journal of Agriculture and Environment 1:260264.

Miliauskas, G., Venskutonis, P.R., Beek, T.A. 2004. 
Screening of radical scavenging activity of some medicinal and aromatic plant extracts. Food Chemistry 85: 231-237.

Morav $\square$ evi $\square$, D., Bjeli $\square$, V., Savi $\square$, D., Gvozdanovi $\square$ Varga, J., Beatovi $\square$, D., Jela $\square$ i $\square$, S., Zari $\square$, V. 2011. Effect of plant density on the characteristics of photosynthetic apparatus of garlic (Allium sativum var.vulgare L.). African Journal of Biotechnology 10: 15861-15868.

Muro, J., Ignacio, I., Lamsfus, C., Fernandez Militino, A. 2000. Effect of defoliation on garlic yield. Scientia Horticulturae 86: 161-167.

Nyakudya, I.W., Stroosnijder, L., 2014. Effect of rooting depth, plant density and planting date onmaize (Zea mays L.) yield and water use efficiency in semi-arid Zimbabwe: Modelling with qua Crop. Agricultural Water Management 146: 280-296.

Olfati, J.A., Peyvast, Gh., Sanavi, M., Salehi, M., Mahdipour, M., 2010. Effect of defoliation on the yield and quality of garlic. Journal of Herbs, Spices \& Medicinal Plants 16: 31-37.

Stahlschmidt, O., Cavagnaro, J.B., Borgo, R., Burba, J.L., Galmarini, C.R., 1997. Influence of planting date and seed cloves size on leaf area and yield of two garlic cultivars (Allium sativum L.). Acta Horticulturae 433: 519-525.

Singleton, V.L., Rossi, J.A., 1965. Colorimetry of total phenolics with phosphomolybdicphosphotungstic acid reagent. American Journal of Enology and Viticulture 16: 144-158.

Wang, Z., Fu, J., He, M., Yin, Y., Cao, H., 1997. Planting density effects on assimilation and partitioning of photosynthates during grain filling in the late-sown wheat 33: 199-204.

Zhang, S., Liao, X., Zhang, C., XU, H., 2012. Influences of plant density on the seed yield and oil content of winter oilseed rape (Brassica napus L.). Industrial Crops and Products 40: 27-32. 